\title{
FRAP Experiments Show Pectate Lyases Promote Pollen Germination and Lubricate the Path of the Pollen Tube in Arabidopsis thaliana.
}

\author{
Youssef Chebli ${ }^{1}$ and Anja Geitmann ${ }^{1}$ \\ ${ }^{1}$ Department of Plant Science, Faculty of Agriculture and Environment Sciences, McGill University, \\ Sainte-Anne-de-Bellevue, Canada
}

The pollen tube is a cellular cylindrical apical growing protuberance formed by the pollen grain upon contact with the stigma, the female organ of the flower [1]. The pollen tube's main purpose is to ensure sexual reproduction in plants by delivering the sperm cells to the female gametophyte. To be able to accomplish this task, the elongating pollen tube has to emerge from the pollen grain by breaking through its thick wall and it has to penetrate the pistillar tissue without precociously bursting. Both situations require the loosening of cell wall material - during germination the pollen intine proper has to yield and during pollen tube elongation the apoplast of the transmitting tract needs to be softened to allow for pollen tube passage [2-3].

Here we investigate the putative role of the pectin digesting enzymes pectate lyases (PLs) in these processes. Using mutant analysis, microscopical observations of fluorescently tagged PLs combined with fluorescence recovery after photo-bleaching (FRAP) experiments and immuno-detection, we show that PLs are required for intine loosening during the first steps of pollen tube germination (Figure 1). During pollen tube elongation, PLs are secreted by the pollen tube into transmitting tract, likely contributing to the digestion and loosening of the pollen tube path.

Our data provide direct evidence for the long-standing assumption that pollen tubes extrude enzymes to the extracellular space with the likely purpose to interact with the transmitting tissue.

\section{References:}

[1] Y Chebli and A Geitmann, Functional Plant Science and Biotechnology, 1 (2007), p. 232.

[2] Y Chebli, J Kroeger and A Geitmann, Molecular Plant 6 (2013), p. 1037.

[3] Y Chebli et al, Plant Physiology 160 (2012), p. 1940.

[4] The authors acknowledge funding from the Fonds Québécois de la Recherche sur la Nature et les Technologies, the Natural Science and Engineering Research Council and the Canadian Foundation for Innovation. 


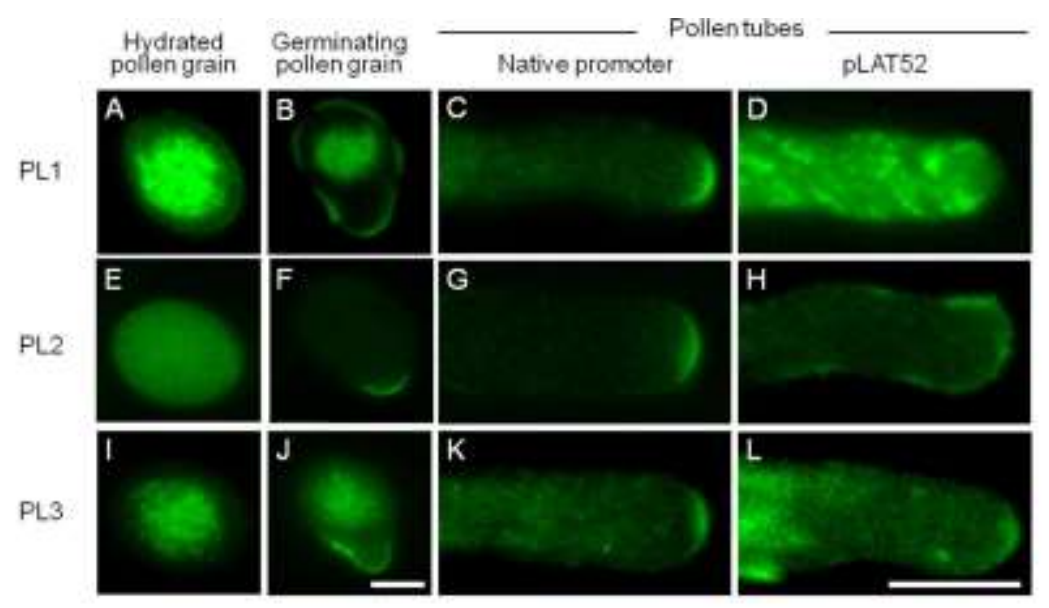

Figure 1. Localization of PLs in pollen grains and tubes. Single confocal sections of hydrated pollen grains (A,E,I), germinating pollen grains (B,F,J), and pollen tubes (C,D,G,H,K,L) expressing fluorescently tagged pectate lyases PL1 (A,B,C,D), PL2 (E,F,G,H) and PL3 (I,J,K,L) under the native promoter $(A, B, C, E, F, G, I, J, K)$ or pLAT52 (D,H,L). Scale bar in J is the same for all pollen grains $(A, B, E, F, I, J)$ and scale bar in $L$ is the same for all pollen tubes $(C, D, G, H, K, L)$. Scale bars $=10 \mu \mathrm{m}$. 